\title{
LIPID MAPS Websites
}

\section{Public website: http://www.lipidmaps.org/}

Development website: http://

dev.lipidmaps.org/ 


\section{Bridge A: LIPID MAPS Networks}

- Reconstruct Proteomic Networks using integration of lipid data from lipidomics laboratories, transcriptional data from Genomic Technologies Laboratory and legacy knowledge.

- Develop methods for quantitative analysis of lipid-proteome networks 


\section{Steps towards reconstruction}

\section{-Establish the parts lists database}

- lipid proteome database

- lipids database

- cell transcriptome database 


\section{Steps towards reconstruction}

-Develop integration and analysis platforms

- statistical analysis of data

- transcriptome analysis

- lipid network analysis 


\section{Steps towards reconstruction}

-Develop interactive pathway building, editing and manipulating tools

- pathway GUI and interaction system

- Integration of GUI with data and databases

- preliminary pathway analysis tools 


\section{Lipid Proteome Database} Overview

- Protein List based on GO and KEGG

- Annotations derived from SWISSPROT, KEGG, GenBank

- Object-Relational Database (Oracle) 


\section{Lipid Proteome Database Schema}

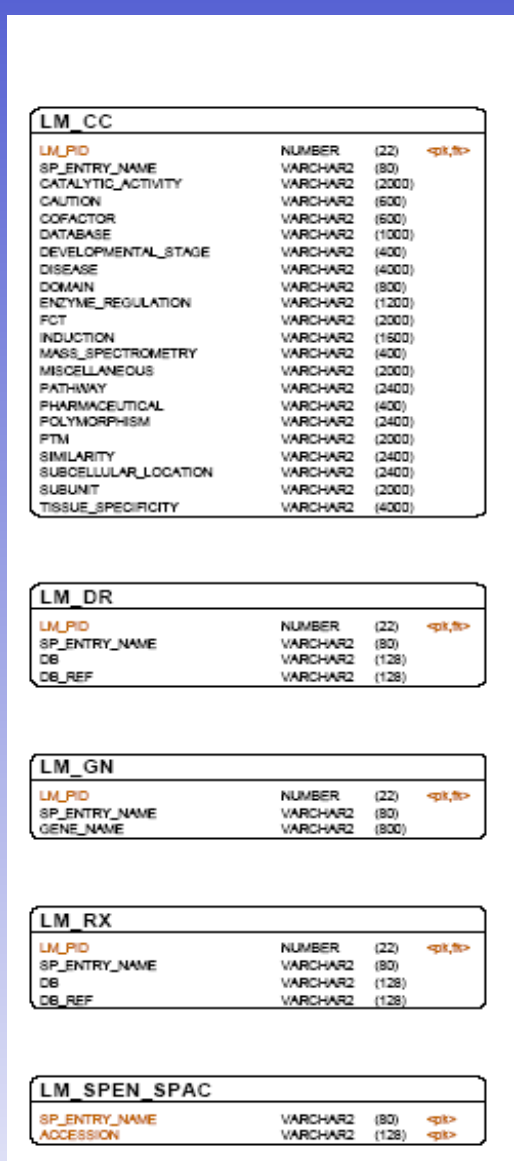

LIPID MAPS Protein Database

\begin{tabular}{|c|c|c|}
\hline LM_PROTEIR & & \\
\hline 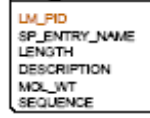 & 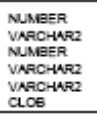 & 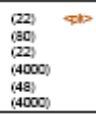 \\
\hline LM_PROTEIN & & \\
\hline $\begin{array}{l}\text { MPID } \\
\text { SEOUENCE }\end{array}$ & $\begin{array}{l}\text { NUMEER } \\
\text { COOG }\end{array}$ & $\begin{array}{l}(222) \\
(4000)\end{array}$ \\
\hline LM_PROP & & \\
\hline $\begin{array}{l}\text { LUPFD } \\
\text { LENSTH } \\
\text { DESCRIPTION } \\
\text { MO_WTT }\end{array}$ & $\begin{array}{l}\text { NUMBER } \\
\text { NAMEER } \\
\text { WNACHAR2 } \\
\text { NAACHAP2 }\end{array}$ & $\begin{array}{l}(22)> \\
(22) \\
(4000) \\
(48)\end{array}$ \\
\hline
\end{tabular}
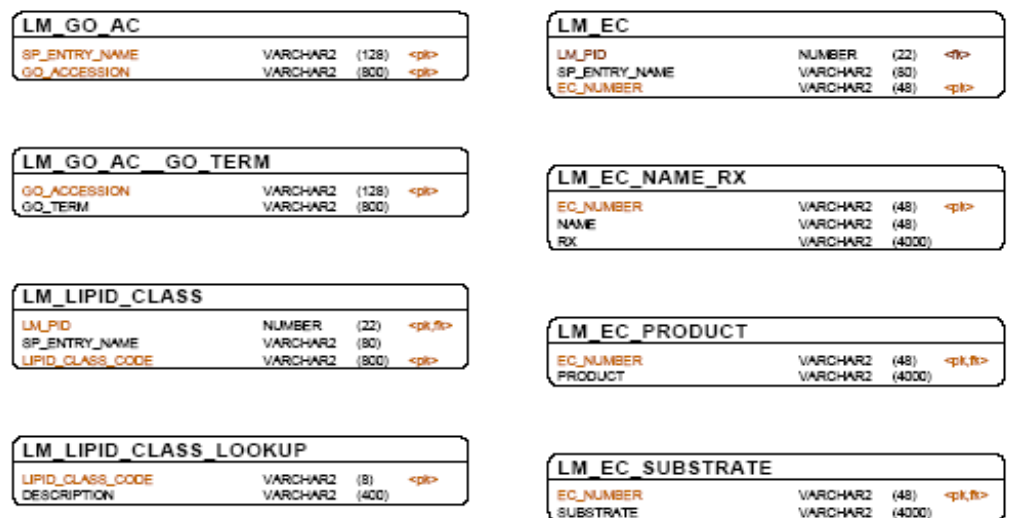

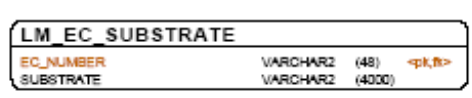
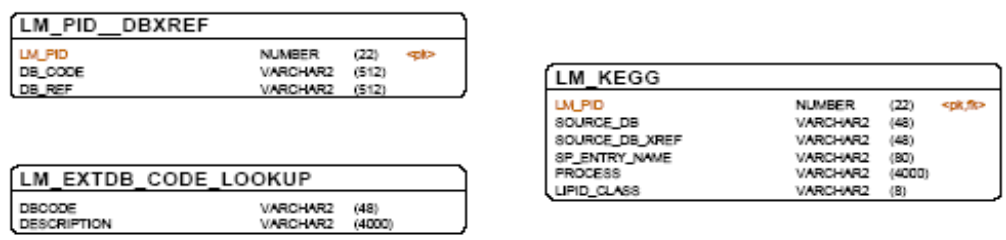

PROCESS
AFID_CWSB

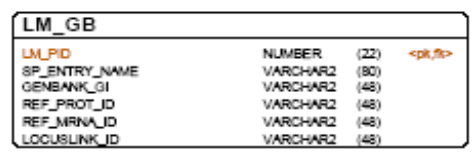




\section{Lipid Proteome Database Statistics}

\begin{tabular}{|l|l|}
\hline Total proteins & 1460 \\
\hline Mouse & 690 \\
\hline Human & 771 \\
\hline $\begin{array}{l}\text { Annotated with Lipid class } \\
\text { association }\end{array}$ & 760 \\
\hline Annotated with KEGG Pathway & 760 \\
\hline KEGG Pathways represented & 109 \\
\hline
\end{tabular}




\section{Lipid Proteome Database Associated Lipid Categories}

\begin{tabular}{|l|l|}
\hline Fatty Acids / Eicosanoids & 84 \\
\hline Glycolipids & 12 \\
\hline Glycerophospholipids & 42 \\
\hline Isoprenoids & 4 \\
\hline Lipid Metabolism & 121 \\
\hline Neutral Lipids & 14 \\
\hline Sphingolipids & 37 \\
\hline Sterols & 85 \\
\hline
\end{tabular}




\section{Lipid Proteome Database Access to Development Version}

\section{http://dev.lipidmaps.org/cgi-bin/protein.cgi}

Username " personnel"

Password " LM5001" 


\begin{tabular}{|c|c|c|c|c|c|c|}
\hline Google. & $\checkmark$ & Search Web & -3 & 氖 3 blocked & 圆AutoFill & 2 Options \\
\hline
\end{tabular}

\section{0}

-upmos:-0 LIPID Metabolites And Pathways Strategy $00^{\circ}$

Protocols

Home

\section{Lipid Categories}

- Fatty Acyls

- Glycerolipids

- Glycerophospholipids

- Sphingolipids

- Sterol Lipids

- Prenol Lipids

- Saccharolipids

- Polyketides

Site Map

Contact

\section{LIPID MAPS Goals}

Separate and detect all of the lipids in a specific cell and discover and characterize any novel lipids that may be present.

Quantitate each of the lipid metabolites present and quantitate the changes in their levels and location during cellular function.

Define the biochemical pathways for each lipid and develop lipid maps which define the interaction networks.
Browse and Search LIPID MAPS Databases LIPID VIAPS IS Tunded

by an National Institute of General Medical Sciences (NIGMS) Glue Grant

NIGMS Press Release

Participating

Institutions and

Researchers

UCSD Press Release 


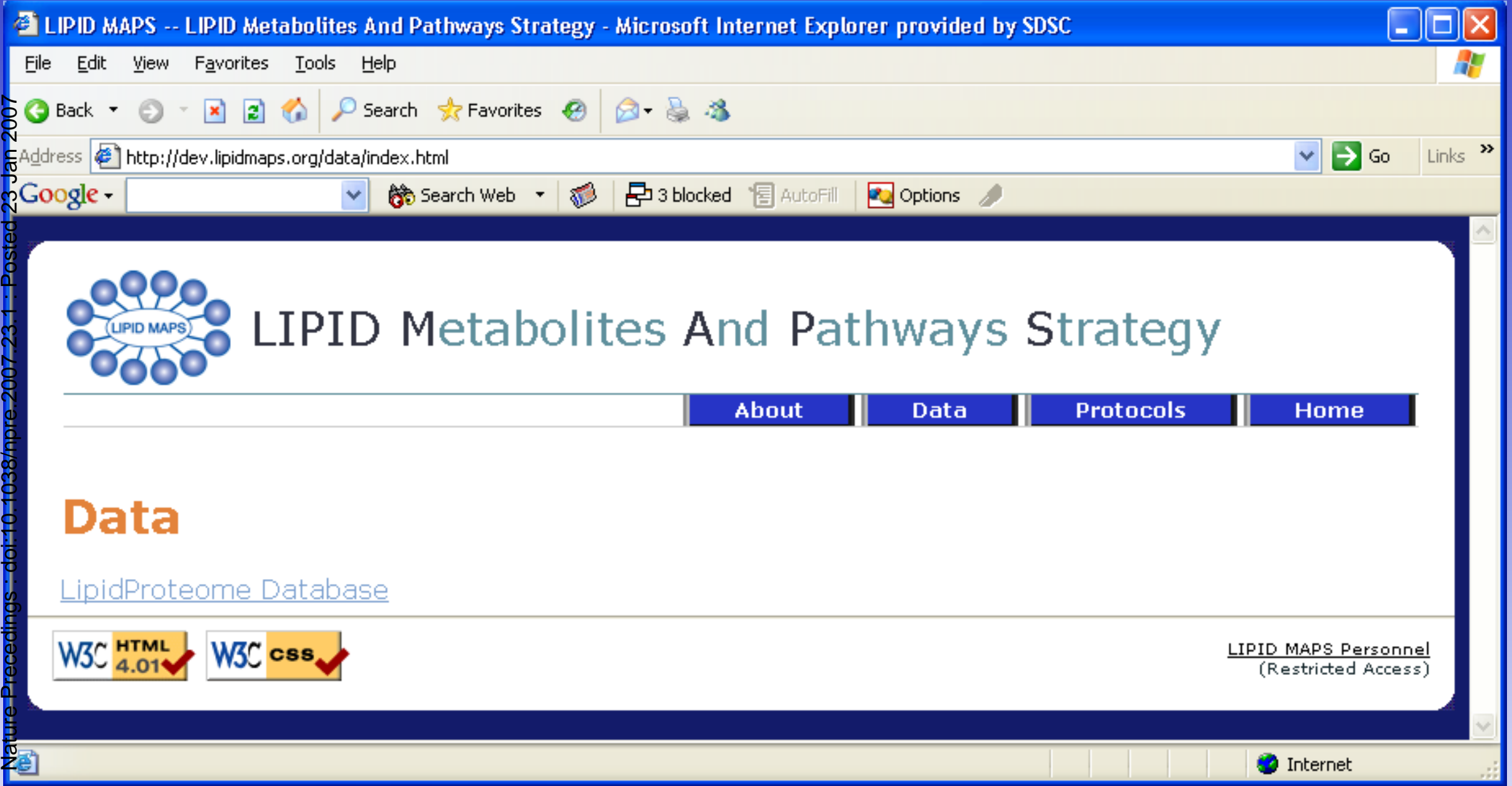




\section{LIPID MAPS Lipid Proteome GUI Overview}

\section{Basic Search (Browse)}

\section{Advanced Search}

\section{Summary Results}

(default is all 1460 records)

Record Details 
TI LIPID MAPS -- LIPID Metabolites And Pathways Strategy - Microsoft Internet Explorer provided by SDSC

Eile Édit view Fagvorites Iools Help

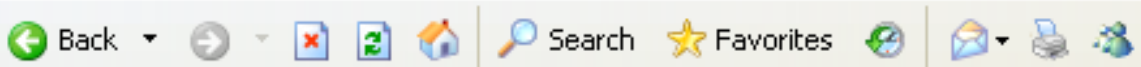

Address hettp://dev.lipidmaps.org/cgi-bin/protein.cgi

$\checkmark \rightarrow$ Go Links $》$
Google.
$\checkmark$ Search web
- B B blocked
圆 ButoFill
Options

\section{LIPID Metabolites And Pathways Strategy}

\section{About}

Data

Protocols

Home

\section{Lipid Proteome Database}

Browse Advanced Search

\begin{tabular}{|c|c|c|}
\hline$\square$ Lim & searc & -- Please Select -- \\
\hline & & - Please Select -- \\
\hline Displa & :5000 & $\begin{array}{l}\text { Fatty acids/Eicosanoids (FA) } \\
\text { Glycolipids (GL) }\end{array}$ \\
\hline Reset & Cancel & $\begin{array}{l}\text { Glycerophospholipids (GP) } \\
\text { Isoprenoids (IP) }\end{array}$ \\
\hline & & $\begin{array}{l}\text { Lipid metabolism (LM) } \\
\text { Neutral Lipids (NL) }\end{array}$ \\
\hline & L & $\begin{array}{l}\text { Sphingolipids (SP) } \\
\text { Sterols (ST) }\end{array}$ \\
\hline
\end{tabular}

LIPID MAPS Personnel (Restricted Access) 


\section{Advanced Search}

\section{Basic Search}

\section{Include data from: O human \\ Omouse $\odot$ both mouse and human}

은 $\square$ Limit search to proteins associated with the lipid class -- Please Select -$\checkmark$

तु $\square$ Limit search to proteins records

ल containing ANY of the words $\checkmark$

Oin any of the search fields selected below

今sequence Properties

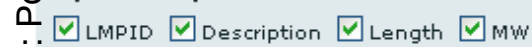

-Swissprot Annotations

ஸे $\square$ swissProt Entry Name $\square$ Function $\square$ post Translational Modification

今 $\square$ Description $\square$ Length $\square$ subcellular Location

\ $\square$ Domain

$\square$ Pathway

Q⿻丷木大KEG Annotations

듬 ${ }_{\text {KEGG Pathway }}$

兵

$\mathscr{m}_{\text {Enzymes, Substrates, Products, Reactions }}$

$\stackrel{ }{\square} \square$ EC Number $\square$ substrate $\square$ product $\square$ Reaction $\square$ Reaction Name

으

$\stackrel{\div}{\frac{\pi}{0}}$

O. *Fields to display in results summary

s

음

$\square$ Function

$\square$ EC Number

Species

$\square$ Length

$\square$ substrate

\Lipid Category

$\square \mathrm{Mw}$

$\square$ KEGG Pathway

$\frac{\square}{(\infty} \square$ swissprot Entry Name $\square$ post Translational Modification $\square$ product

$\stackrel{\oplus}{J} \square$ Description $\square$ subcellular Location $\square$ Reaction

艺

Z

("Full record details are accessable from the summary page)

Display:5000 $\vee$ records per page.

\begin{tabular}{|l|l|}
\hline Search Reset Cancel \\
\hline
\end{tabular} 


\section{LIPID Metabolites And Pathways Strategy}

\section{Lipid Proteome Database}

Records 1 - 1460 of 1460

New Search

Sort by: LMPID $\quad v$ ( $\odot$ ascending Odescending ) Sort

Display selected records Display sequences in FASTA format

\begin{tabular}{|c|c|c|c|}
\hline LMPID & Species & $\begin{array}{c}\text { Lipid } \\
\text { Category } \\
\text { leqend }\end{array}$ & $\begin{array}{l}\text { SwissProt } \\
\text { Entry Name }\end{array}$ \\
\hline
\end{tabular}

\section{Description}

$\square 1$ human 1432_HUMAN

14-3-3 protein zeta/delta (Protein kinase C inhibitor protein-1) (KCIP-1) (Factor activating exoenzyme S) (FAS)

Serine/threonine protein phosphatase $2 A, 65 \mathrm{KDA}$ regulatory subunit $\mathrm{A}$, alpha isoform

$\square$ 2 human SP 2AAA_HUMAN
(PP2A, subunit A, PR65-alpha isoform) (PP2A, subunit A, R1-alpha isoform) (Medium tumor antigen-associated $61 \mathrm{KDA}$ protein)

Serine/threonine protein phosphatase $2 \mathrm{~A}, 65 \mathrm{kDa}$ regulatory subunit $\mathrm{A}$, beta isoform ( $\mathrm{PP} 2 \mathrm{~A}$, subunit A, PR65-beta isoform) (PP2A, subunit A, R1-beta isoform)

3 beta-hydroxysteroid dehydrogenase/delta 5-->4-isomerase type I (3Beta-HSD I)

(Trophoblast antigen FDO161G) [Includes: 3-beta-hydroxy- delta(5)-steroid dehydrogenase (EC 1.1.1.145) (3-beta-hydroxy-5-ene steroid dehydrogenase) (Progesterone reductase); Steroid delta-isomerase (EC 5.3.3.1) (Delta-5-3-ketosteroid isomerase)]

3 beta-hydroxysteroid dehydrogenase/delta 5-->4-isomerase type II (3Beta-HSD II) [Includes: 3-beta-hydroxy-delta(5)-steroid dehydrogenase (EC 1.1.1.145) (3-beta-hydroxy5 -ene steroid dehydrogenase) (Progesterone reductase); Steroid delta-isomerase (EC 5.3.3.1) (Delta-5-3-ketosteroid isomerase)]

Lactosy/ceramide 4-alpha-galactosyltransferase (EC 2.4.1.228) (Alpha- 1.4galactosyltransferase) (UDP-galactose:beta-D-galactosyl-beta1-R 4- alpha-Dgalactosyltransferase) (Alpha-1,4-N-acetylglucosaminyltransferase) (Alpha4Gal-T1) (Globotriaosylceramide synthase) (Gb3 synthase) (CD77 synthase) (P1/Pk synthase)

$\square \underline{6}$ human SP A4GT_HUMAN

Potential phospholipid-transporting ATPase IC (EC 3.6.3.1) (Familial intrahepatic cholestasis type 1) (ATPase class I type BB member 1)

\begin{tabular}{|l|l|l|l}
\hline$\square \underline{z}$ & human & LM & ABB1_HUMAN \\
\hline$\square \underline{g}$ & human & & ASAD_HUMAN \\
\hline$\square \underline{g}$ & human & LM & AACT_HUMAN \\
\hline$\square \ldots$ &, & FA & $\ldots \ldots \ldots \ldots$ \\
\hline
\end{tabular}

Arylacetamide deacetylase (EC 3.1.1.-) (AADAC)

Alpha-1-antichymotrypsin precursor (ACT) 


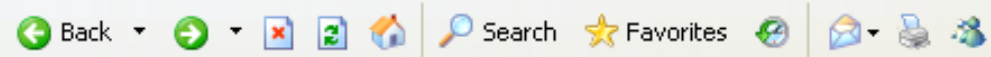

Address hittp://dev,lipidmaps.org/cgi-bin/protein.cgi?form=advanced

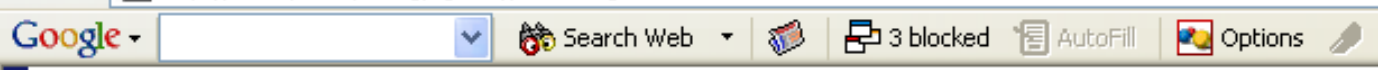

\section{Advanced Search Basic Search}

Oิ Include data from: Ohuman $\bigcirc$ mouse $\odot$ both mouse and human

$\underset{\widetilde{N}}{\stackrel{C}{\sim}} \square$ Limit search to proteins associated with the lipid class -- Please Select --

$\square$ Limit search to proteins records

\begin{tabular}{ll|l} 
D. & containing the PHRASE \\
if & containing ANY of the words
\end{tabular}

.. containing ALL of the words

- containing the PHRASE

ஸे $\square$ LMPID $\square$ Description $\square$ Length $\square$ Mw

N SwissProt Annotations

尺 $\square$ swissProt Entry Name $\square$ Function $\square$ Post Translational Modification

흘 $\square$ Description $\square$ Length $\square$ subcellular Location

『Domain $\square$ Pathway

\% KEGG Annotations

Oั $\square$ KEGG Pathway

Enzymes, Substrates, Products, Reactions

$\therefore \square$ EC Number $\square$ substrate $\square$ Product $\square$ Reaction $\square$ Reaction Name

*Fields to display in results summary

$\begin{array}{lll}\square \text { LMPID } & \square \text { Function } & \square \text { EC Number } \\ \square \text { Species } & \square \text { Length } & \square \text { substrate } \\ \square \text { Lipid Category } & \square \text { Mw } & \square \text { KEGG Pathway }\end{array}$

$\square$ SwissProt Entry Name $\square$ Post Translational Modification $\square$ product

ఐDescription $\square$ Subcellular Location $\square$ Reaction

ఐDomain $\square$ Pathway Reaction Name

("Full record details are accessable from the summary page) 


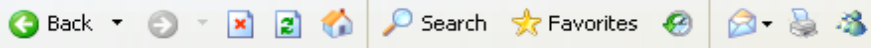

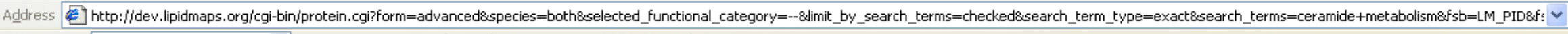

Google -

$\checkmark$ Search Web -2 号 3 blocked 圆 AutoFill 2 Options

\section{LIPID Metabolites And Pathways Strategy}

\section{Lipid Proteome Database}

Records $1-8$ of 8

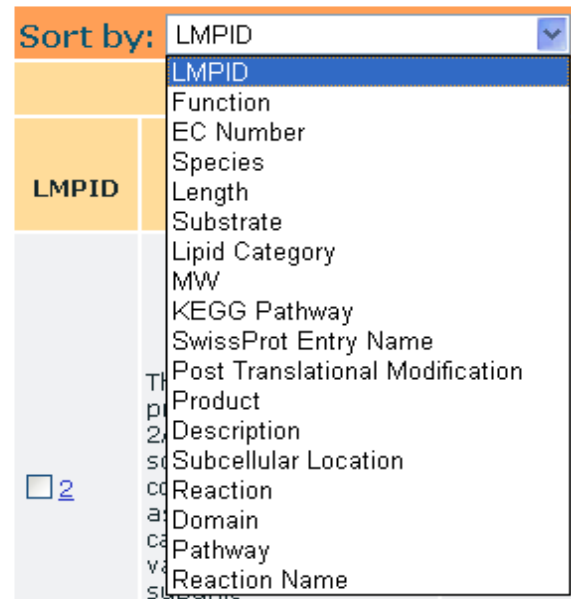

( $\odot$ ascending Odescending ) Sort

\begin{tabular}{|c|c|c|c|c|c|c|}
\hline & & & & \multicolumn{3}{|c|}{ Display selected records } \\
\hline Species & Length & Substrate & $\begin{array}{c}\text { Lipid } \\
\text { Category } \\
\text { leqend }\end{array}$ & MW & $\begin{array}{l}\text { KEGG } \\
\text { Pathway }\end{array}$ & $\begin{array}{l}\text { SwissProt } \\
\text { Entry Name }\end{array}$ \\
\hline
\end{tabular}

human 588

$\mathrm{SP}$

65092 ceramide

2AAA_HUMAN human 601

protein subunit of

protein phosphatase

$2 \mathrm{~A}$ serves as a

scaffolding molecule to

coordinate the

assembly of the

catalytic subunit and a

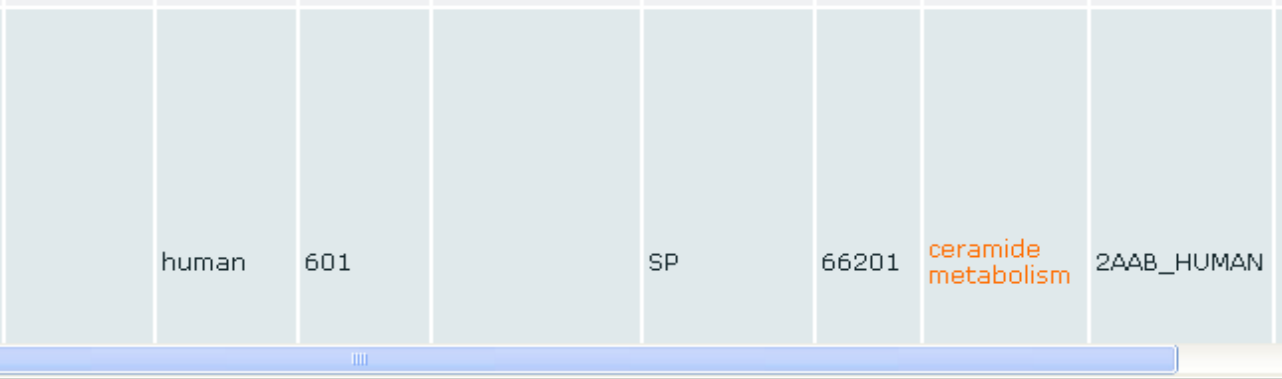

\begin{tabular}{|l|l}
\hline \multicolumn{2}{|c|}{ Display sequences in FASTA format } \\
\hline $\begin{array}{c}\text { Post Translational } \\
\text { Modification }\end{array}$ & Product \\
\hline
\end{tabular}

Serine/tr

protein

phospha

$65 \mathrm{KDA}$

subunit

isoform (

subunit alpha iso
(PP2A, s

R1-alpha

(Medium

antigen-

associate

KDA prot

Serine/th

protein

$65 \mathrm{kDa}$.

subunit $A$

isoform?

isoform (

beta isof 


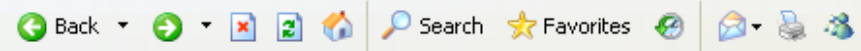

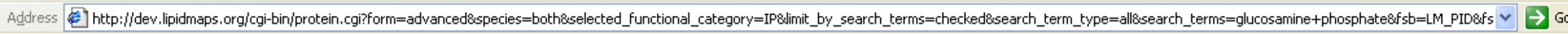
Google -

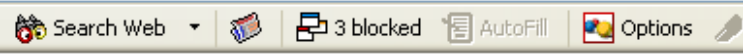

\section{Advanced Search}

\section{Basic Search}

Include data from: Ohuman Omouse $\odot$ both mouse and human

O $\square$ Limit search to proteins associated with the lipid class -- Please Select -

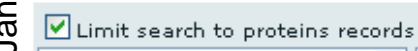

containing the PHRASE $\vee$ ceramide metabolism

in any of the search fields selected below

Sequence Properties

$\triangle \square$ LMPID $\square$ Description $\square$ Length $\square$ Mw

- swissprot Annotations

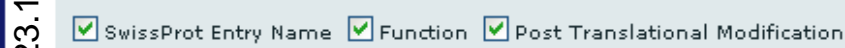

N Description $\square$ Length $\square$ subcellular Location

四Domain $\square$ Pathway

NEGG Annotations

는 $\square$ KEGG Pathway

Enzymes, substrates, Products, Reactions

品 $\square$ EC Number $\square$ substrate $\square$ Product $\square$ Reaction $\square$ Reaction Name

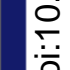

음 *Fields to display in results summary
$\square$ Length
$\square$ Substrate
$\checkmark$ Lipid Category
$\square$ Mw
$\square$ KEGG Pathway
ป $\square$ swissprot Entry Name $\square$ post Translational Modification $\square$ Product
(1) Description $\square$ Subcellular Location $\square$ Reaction
$\square$ Domain
$\square$ Reaction Name

$\square$ Function

$\square$ EC Number

("Full record details are accessable from the summary page)

Display: $50 \vee$ records per page.

Search Reset Cancel 


\section{File Edit View Fagvorites Iools Help

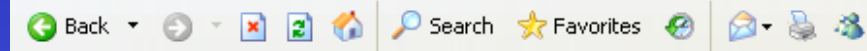

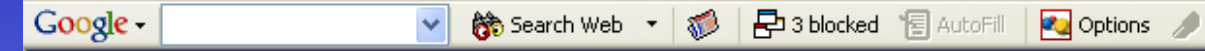 \\ 0990 $060^{\circ}$}

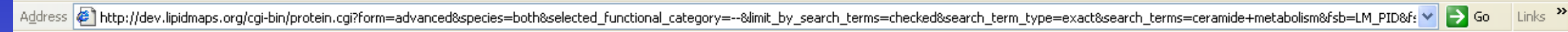

\section{Lipid Proteome Database}

Records $1-8$ of 8

New Search

\begin{tabular}{|c|c|}
\hline Sort by: & LMPID \\
\hline & LMPID \\
\hline & EC Number \\
\hline & Species \\
\hline LMPID & Lipid Category \\
\hline & KEGG Pathway \\
\hline & $\begin{array}{l}\text { SwissProt Entry Name } \\
\text { Product }\end{array}$ \\
\hline & $\begin{array}{l}\text { Description } \\
\text { Subcellular Location }\end{array}$ \\
\hline$\square \underline{2}$ & Reaction \\
\hline
\end{tabular}

$\checkmark$ ( $\odot$ ascendine ruesceriumy $:$ Sort

\begin{tabular}{|c|c|c|c|c|}
\hline & \multicolumn{3}{|c|}{ Display selected records } & Display sequences in FA \\
\hline Substrate & $\begin{array}{l}\text { Lipid } \\
\text { Category } \\
\text { leqend }\end{array}$ & $\begin{array}{c}\text { KEGG } \\
\text { Pathway }\end{array}$ & $\begin{array}{l}\text { SwissProt } \\
\text { Entry Name }\end{array}$ & Product \\
\hline
\end{tabular}

Subcellular Location

ceramide 2AAA_HUMAN

$\square \underline{3}$

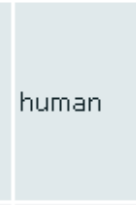

$\square \underline{72}$

3.5.1.23 human $\mathrm{N}$

$\begin{array}{ll}\mathrm{N}- & \mathrm{FA} \\ \text { acylsphingosine } & \mathrm{SP}\end{array}$

P

ceramide

metabolism

ASAH_HUMAN carboxylate

sphingosine

$\mathrm{ADP}$

\section{Description}

Serine/threonine protein phosphatase $2 \mathrm{~A}, 65 \mathrm{KD}$

regulatory subunit $A$

alpha isoform (PP2A,

subunit A, PR65-alpha

isoform) (PP2A, subunit $A$,

R1-alpha isoform)

(Medium tumor antigen-

associated $61 \mathrm{KDA}$

protein)

Serine/threonine protein phosphatase $2 \mathrm{~A}, 65 \mathrm{kDa}$

regulatory subunit $A$

regulatory subunit.

subunit A, PR65-bet

isoform) (PP2A, subunit A,

R1-beta isoform

Acid ceramidase

precursor (EC 3.5.1.23)

(Acylsphingosine

deacylase) ( $\mathrm{N}$ -

acrlsphingosine

acysphingosine

amidohydrolase) (AC)

(Putative $32 \mathrm{KDA}$ heart

protein) (PHP32)

Ceramide kinase (EC

2.7.1.138) Cytoplasmic and

(Acylsphingosine kinase)

(hCERK) (Lipid kinase 4)

membrane-

associated

Subcellular

Location

Reaction

(LK4)

Protein FAN (Factor $\mathrm{H} 2 \mathrm{O}=$ a carboxylate + sphingosine

$\mathrm{ATP}+$ ceramide $=\mathrm{ADP}+$ ceramide 1-phosphate 


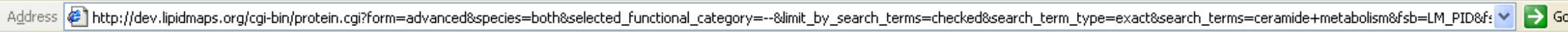
Google -

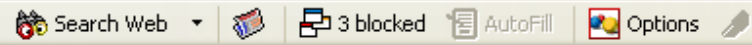

\section{0}

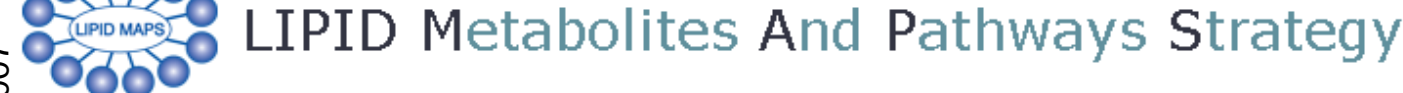

ㄴ.

on

Records 1 - 8 of 8

New Search

Sort by: LMPID

$\checkmark(\odot$ ascendin, ouescenainy)

ํ.

Display selected records

Display sequences in FASTA format

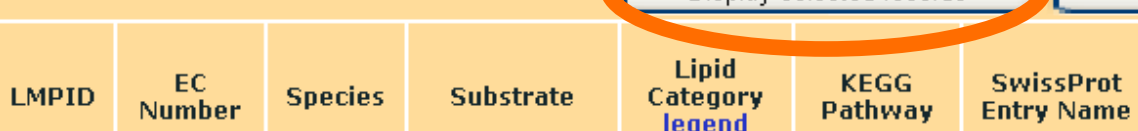
Description

Subcellular Location

Reaction

Serine/threonine protein phosphatase 2A, 65 KDA regulatory subunit $A$ alpha isoform (PP2A, subunit A, PR65-alpha Isoform) (PP2A, subunit $A$ R1-alpha isoform) (Medium tumor antigenassociated $61 \mathrm{KDA}$ protein)

Serine/threonine protein phosphatase $2 \mathrm{~A}, 65 \mathrm{kDa}$ regulatory subunit $A$,

beta isoform (PP2A,

subunit A, PR65-beta

isoform) (PP2A, subunit A

R1-beta isoform)

Acid ceramidase

precursor (EC 3.5.1.23)

(Acylsphingosine

deacylase) ( $\mathrm{N}$ -

acylsphingosine

amidohydrolase) (AC)

(Putative 32 KDA heart

protein) (PHP32)

Ceramide kinase (EC

2.7.1.138)

ADP

(Acylsphingosine kinase)

(hCERK) (Lipid kinase 4)

(LK4)
$\mathrm{N}$-acylsphingosine +

Lysosomal $\quad \mathrm{H} 2 \mathrm{O}=\mathrm{a}$ carboxylate sphingosine

Cytoplasmic and $\mathrm{ATP}+$ ceramide $=\mathrm{ADP}+$ membraneassociated ceramide 1-phosphate 

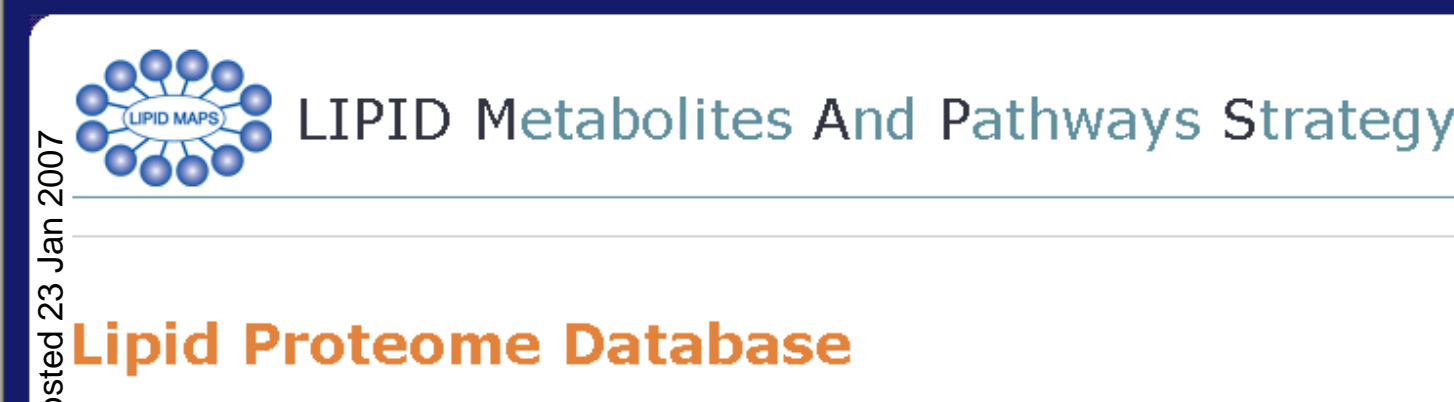

\section{(5) \\ Selected complete database records:}

กั

LMPID

Description

New Search

Serine/threonine protein phosphatase 2A,65 KDA regulatory subunit A, alpha isoform (PP2A, subunit A, PR65-alpha isoform) (PP2A, subunit A, R1-alpha isoform) (Medium turnor antigen61 KDA protein)

Serine/threonine protein phosphatase $2 \mathrm{~A}, 65 \mathrm{kDa}$ regulatory subunit $\mathrm{A}$, beta isoform (PP2A, subunit A, PR65-beta isoform) (PP2A, subunit A, R1-beta isoform)

Acid ceramidase precursor (EC 3.5.1.23) (Acylsphingosine deacylase) ( $\mathrm{N}$-acylsphingosine amidohydrolase) (AC) (Putative 32 KDA heart protein) (PHP32)

\section{LMPID 2}

\section{Record Overview}

LMPID

2

Species

human

Description

Lipid Category

Serine/threonine protein phosphatase 2A, 65 KDA regulatory subunit A, alpha isoform (PP2A, subunit A, PR65-alpha isoform) (PP2A, subunit A, R1-alpha isoform) (Medium tumor antigen-associated 61 KDA protein)

EC Number

Z $\mathrm{MW}$

Sphingolipids (SP)

Length

65092

Sequence

588

<view>

\section{SWISS PROT Annotations}

SwissProt Entry

Name

Catalytic Activity 
File Edit Yiew Favorites Iools $\underline{\text { Help }}$

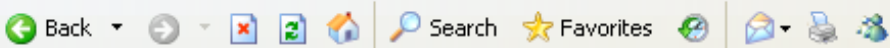

Address hittp://www.genome.jp/dbget-bin/get_pathway?org_name=hsa\&mapno=00600

$\rightarrow$ Go Links $\gg$

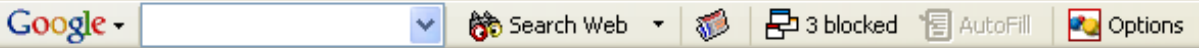

TS Glycosphingolipid metabolism - Homo sapiens

[ LinkDB search | Reference list | Pathway menu ]

Homo sapiens

$\checkmark$ Go Next

GLYCOSPHINGOLIPID METABOLISM

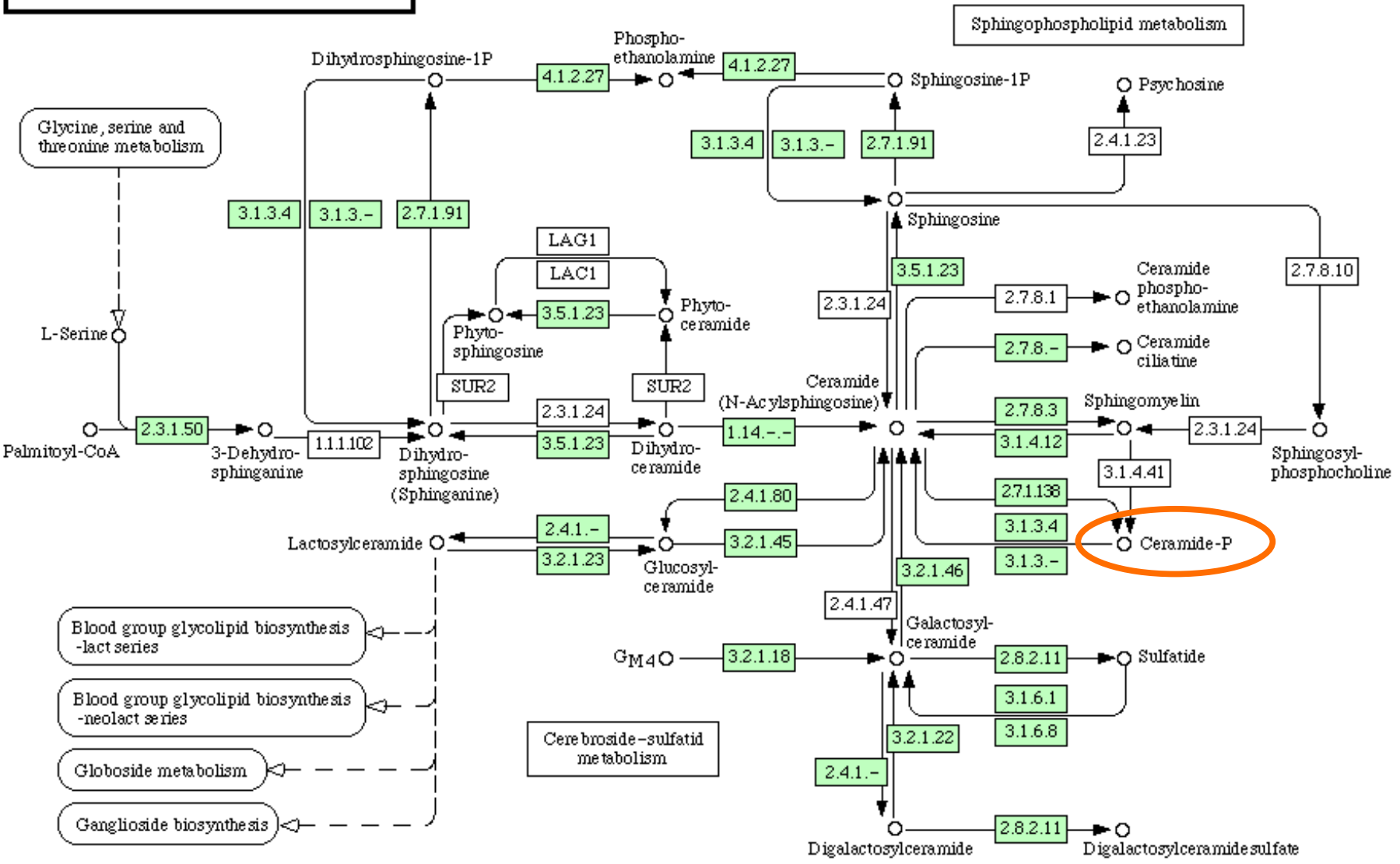




\section{Eile Édit Yiew Fạvorites Iools Help

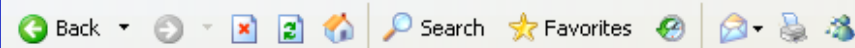 Google. \\ Search Web - B 3 blocked 圆AutoFill \\ 0990

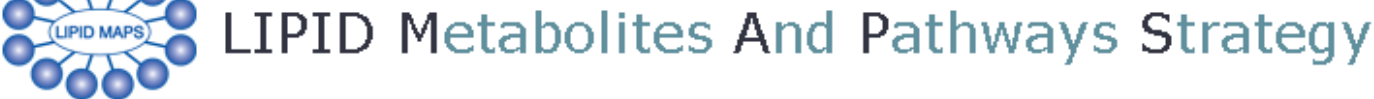

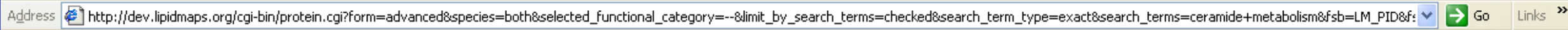

\section{Lipid Proteome Database}

Records $1-8$ of 8

New Search

\begin{tabular}{l|l} 
Sort by: & LMPID \\
\hline LMPID & \begin{tabular}{|l} 
LMPID \\
EC Number \\
Species \\
Substrate \\
Lipid Category \\
KEGG Pathway \\
SwissProt Entry Name \\
Product \\
Description \\
Subcellular Location \\
Reaction
\end{tabular} \\
\hline$\underline{2}$
\end{tabular}

$\checkmark$ ( $\odot$ ascending Odescending) Sort

$\square \underline{2}$ Reaction

Substrate

Display selected records

10

Display sequences in FASTA format

KEGG

ategory

leqend

Pathway

SwissProt
Entry Name

ceramide

2AAA_HUMAN
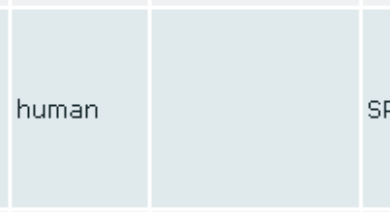

ceramide

2AAB_HUMAN

\section{$\square \underline{72}$}

3.5.1.23 human

$\mathrm{H} 2 \mathrm{O}$
$\mathrm{N}-$

FA

acylsphingosine SP

ceramide CEK1_HUMAN $\begin{aligned} & \text { ADP } \\ & \text { ceramide 1- }\end{aligned}$ phosphate
Product

Description

Serine/threonine protein phosphatase $2 \mathrm{~A}, 65 \mathrm{KDA}$

regulatory subunit $A$

regulatory subunit $A$

alpha isoform (PP2A,

isoform) (PP2A, subunit A,

R1-alpha isoform)

R1-alpha Isoform)
(Medium tumor antigen-

associated $61 \mathrm{KDA}$

protein)

Serine/threonine protein

phosphatase $2 \mathrm{~A}, 65 \mathrm{kDa}$

regulatory subunit $A_{\text {, }}$

regulatory subunit A,

beta isoform (PP2A,

subunit A, PR65-beta
isoform) (PP2A, subunit A,

R1-beta isoform)

Acid ceramidase

precursor (EC 3.5.1.23)

(Acylsphingosine

deacylase) ( $\mathrm{N}$ -

acrlsphingosine

acylsphingosine

amidohydrolase) (AC)

(Putative 32 KDA heart

protein) (PHP32)

Ceramide kinase (EC

2.7.1.138) Cytoplasmic and

(Acylsphingosine kinase)

(hCERK) (Lipid kinase 4)

(LK4)

Protein FAN (Factor membrane-

\section{Subcellular \\ Location \\ Reaction} $\mathrm{H} 2 \mathrm{O}=$ a carboxylate + sphingosine

associated

$\mathrm{ATP}+$ ceramide $=\mathrm{ADP}+$ ceramide 1-phosphate 


\section{Lipid Proteome Database}

$>$ LM:2|2AM_HUMHN|Serine/threonine protein phosphatase $2 \mathrm{~A}, 65 \mathrm{KDA}$ regulatory ; subunit $\overline{\mathbf{A}}$, alpha isoform (PP2

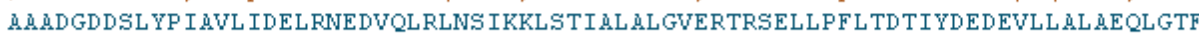
TTLVGGPE YVHCLLPPLESL A TVEE TVVRD KAVESLRA ISHE HSPSDLE AHFVPLVKRL AGGDWF TSRTS ACGLF

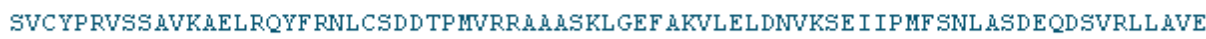
ACVN I AQL L PQEDL E ALVMP TL RQA A E D KSW AVR YNV AD KF TE LQKAVGP I I TKTDLVP AF ONL MKDCE AEVRA A ASHKVKE F CENLS ADCRENV I MSQIL PC I KE LVSD ANQHVKS AL ASV I MGLSP IL GKDNT IE HL L PLF L AQL KDF CPEVRLN I ISNL DCVNEV I G IRQLSQSLL P AVEL A E D A KURVRL A I I YMPLL A GQLGVEF F DE KLNSLCMA WL VD HVY A IRE A A TSNL KKL VE KF GKE W AHAT I IP KVL AMS GD PNYL HRMTTLF C INVLSEVCGQD ITTKHMLP TVL RMA GD PV ANVRFNVA KSL QKI GP IL DNSTLQSEVKP IL E KL TQDQDVDVKYF AQE AL TVLSL A

$>\mathrm{LM}: 3 \mid 2 \mathrm{AMB}$ HUMAN|Serine/threonine protein phosphatase $2 \mathrm{~A}, 65 \mathrm{kDa}$ regulatory

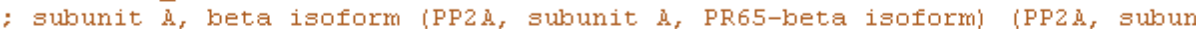
MAG ASELGTGP GA AGGDGDDSL YP I AVL IDEL RNEDVQLRLNS IKKLSTI AL ALGVERTRSELLPFLTDTIYDED EVLL AL AEQL GNF TGLVGGPDF AHCLLPPLENL A TVE E TVVRD KAVESLRQISQE HTPVALE A YFVPLVKRL ASG DWF TSRTS ACGLF SVCYPRASNAVKAE TRQQF RSLCSDD TP NVRR A A ASKLGEF AKVLELDSVKSE IVPLF TSL A SDEQDSVRL AVE ACVS I AQLLSQD DLE TLVMP TL RQẢ AE D KS STRVRYMV AD RF SELQKAMGPKI TLNDL IP AF $O$ NLL KD CE A EVR A A A A HKVKE L GENL P IEDRE TI IMNQIL P Y I KELVSD TNQHVKS AL ASV I MGLST IL GKENT IE HLL PLF L A QL KD ECP DVRLN I ISNL D CVNEV I G IRQLSQSLLP A IVEL AED A KWRVRL A I IE YMPLL A GQL GVEF FDE KLNSLCM TWLVD HVY A IRE A A TNNL MKLVQKF GTE W AONT IVP KVLVM AND PNYL HRMTTLFC INALSE ACG

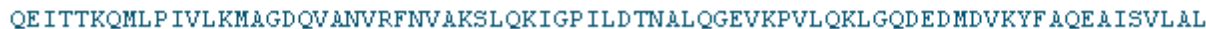
is

$>$ LM:72| מSAH_HUM ; deacylase) (N-acylsphingosine amidohydrolase) (AC) (Putative 32 KD A heart MPGRSCVALVLL AAAVSCAVAOHAPPUTEDCRKSTYPPSGP TYRG AVPWYT INL DLPPYKRWHEL MLDKAPMLKV

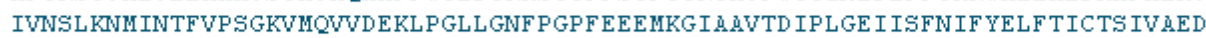
KKGHL I HGRNMD F GVF L GIN INND TWV I TEQL KP L TVNL D F QRNNKTVF KASSF A GYVGML TGF KP GLF SL TLNE RFS INGGYL GILE W IL GKKD AMW I GF L TRTVL ENSTSYE E AKNLL TKTKIL A A AYF I LGGNQSGEGCVITRDRKF SLDVYELD A KQGRWYVVQTNYDRWKHPF FLDDRRTP AKMCLNR TSQEN ISF E TMYDVLSTKPVLNKL TVYTTL ID VTKGQFETYLRDCPDPCIGW 